Preprint typeset in JHEP style - HYPER VERSION

\title{
Brane-world Quantum Gravity
}

\author{
M.D. Maia*, Nildsen Silva ${ }^{\dagger}$ and M.C.B. Fernandes ${ }^{\ddagger}$ \\ Instituto de Física, Universidade de Brasilia, \\ 70919-970, Brasilia, D. F., Brazil
}

\begin{abstract}
The Arnowitt-Deser-Misner canonical formulation of general relativity is extended to the covariant brane-world theory in arbitrary dimensions. The exclusive probing of the extra dimensions makes a substantial difference, allowing for the construction of a non-constrained canonical theory. The quantum states of the brane-world geometry are defined by the Tomonaga-Schwinger equation, whose integrability conditions are determined by the classical perturbations of submanifolds contained in the Nash's differentiable embedding theorem. In principle, quantum brane-world theory can be tested by current experiments in astrophysics and by near future laboratory experiments at Tev energy. The implications to the black-hole information loss problem, to the accelerating cosmology, and to a quantum mathematical theory of four-sub manifolds are briefly commented.
\end{abstract}

Keywords: Quantum Gravity Brane-world Nash Theorem Tomonaga-Schwinger.

*maia@unb.br

${ }^{\dagger}$ nildsen@hotmail.com

${ }^{\ddagger}$ mcezar@fis.unb.br 


\section{Contents}

1. Quantizing the Brane-World

2. Covariant Brane-world Gravity 2

3. Canonical Equations 6

4. Tomonaga-Schwinger Quantum States 8

5. Overview and Perspectives 10

\section{Quantizing the Brane-World}

If gravity is to occupy a significant place in modern physics, it can do so only by being qualitatively different from other fields. As soon as we assume that gravity behaves qualitatively like other fields, we find that it is quantitatively insignificant C.W. Misner (1957)

After analyzing perturbative quantum gravity, Misner reached the interesting conclusion that an effective quantum gravity must have qualities which makes it different from gauge theories [1]. Translating quantitative significance in terms of energy level, Misner's conclusion suggests that the problem of quantization of the gravitational field should be solved concomitantly with the hierarchy problem of the fundamental interactions. In what follows, we apply this criterium to brane-world gravity.

Brane-world gravity is based on a higher dimensional solution of the hierarchy problem. In a seminal paper N. Arkani-Hamed, G. Dvali and S. Dimopolous questioned the currently accepted hypothesis that gravitons are quantitatively relevant only at the Planck scale of energies, essentially because this is an assumption devoid of experimental support. They proposed that the known gauge fields (and hence all ordinary matter) are to be confined within the four-dimensional brane-world, but gravitons can propagate in a higher-dimensional space, the bulk, at the same Tev scale of energies of the gauge fields [2] (For historical papers on the development of the theory see also [3, 4, 5, 6, 0, 8]). According to this view, brane-world gravity is qualitatively distinct from, but it is quantitatively equivalent to the gauge fields of the standard model.

Brane-world gravity predicts the existence of short lived Tev mini black holes, which in principle can be produced at the laboratory by a high energy proton-proton collision, with implications to the black hole information loss problem at the quantum level. The 
proposed experiment is set in Minkowski space-time, but it ends in a Schwarzschild (or Reissner-Nordstrom) space-time [9]. Therefore, the theory supporting this experiment must be compatible with cross sections of the order of the Schwarzschild radius, and also with an explanation on how the original Minkowski space-time deforms into black hole, and back in a short period of time.

Brane-world gravity may also explain the acceleration of the universe (see eg [10] and references therein). In short, due to the presence of the extrinsic curvature, the vacuum in brane-world gravity is richer than the vacuum in general relativity. Besides the cosmological constant, it also contain a conserved geometric tensor built from the extrinsic curvature. Consequently, when studying the quantum fluctuations of such vacuum we may obtain a different estimate for the vacuum energy density as compared with the case of general relativity.

Since most of the current research on brane-world theory is based on models defined in a five-dimensional bulk, using specific coordinates and particular symmetries (see eg [11]), we find it necessary to review in the next section the covariant equations of motion of a brane-world defined in an arbitrary bulk, with an arbitrary number of dimensions. Those equations can be found elsewhere [12, 10], but here we have included some details which are required for the quantum description. Readers who are familiar with this may jump to section 3, where the canonical equations of the brane-world with respect to the extra dimensions are discussed. In section 4 we introduce the Tomonaga-Schwinger equation for the brane-world with respect to the extra dimensions and comment on its integrability.

\section{Covariant Brane-world Gravity}

There are essentially three basic postulates in brane-world theory: (1) The bulk geometry is defined by Einstein's equations; (2) The brane-world is a sub manifold embedded in that bulk ; (3) The gauge fields and ordinary matter are confined to four dimensions, but gravitons propagates along the extra dimensions at Tev energy [2].

The embedding of the brane-world in the bulk plays an essential role on the covariant (that is, model independent) formulation of the brane-world gravity, because it tells how the Einstein-Hilbert dynamics of the bulk is transferred to the brane-world. However, there a are many different ways to embed a manifold into another, classified as local, global, isometric, conformal (or more generally defined by a collineation), rigid, deformable, analytic or differentiable. The choice of one or another depend on what the embedded manifold is supposed to do.

In string theory the action principle is defined on the world-sheets, with additional boundary conditions, so that the embedding is necessarily global. Since the world sheets are 2-dimensional they are all conformally flat and their global embedding is not difficult to achieve. However, if higher-dimensional objects such as p-branes are to be considered, then the global embedding may turn out to be difficult to realize in 10 or even in 11 dimensions 13].

Differently from string theory, the Einstein-Hilbert action in brane-world theory is set on the bulk, which is therefore the primary dynamical object. Furthermore, the embedding 
is locally defined, meaning that the bulk is a local fiber bundle whose fibers are the direct sum of the tangent and normal spaces at each point of the brane-world taken as the base space. If we want to draw a picture, the bulk can be seen as as a locally constructed space around each point of the brane-world.

A local differentiable embedding requires only that the embedding functions are differentiable and regular. This follows from Nash's embedding theorem, an important improvement over the traditional analytic embedding theorems of Janet and Cartan [14, 15], which demand that the embedding functions are represented by convergent positive power series. Furthermore, Nash's theorem shows that any sub manifold can be generated by a continuous sequence of small perturbations of an arbitrarily given sub manifold ${ }^{1}$. Although the theorem was originally demonstrated for the case of an Euclidean bulk, it was later generalized to pseudo Riemannian manifolds [18, 19].

Given a particular Riemannian sub-manifold $\overline{\sigma_{4}}$, its local isometric embedding in a certain bulk $\mathcal{M}_{D}$, is given by $D=4+N$ differentiable and regular embedding maps $\overline{\mathcal{X}}^{A}: \bar{\sigma}_{4} \rightarrow \mathcal{M}_{D}$, such that ${ }^{2}$

$$
\overline{\mathcal{X}}_{, \mu}^{A} \overline{\mathcal{X}}_{, \nu}^{B} \mathcal{G}_{A B}=\bar{g}_{\mu \nu}, \quad \overline{\mathcal{X}}_{, \mu}^{A} \bar{\eta}_{b}^{B} \mathcal{G}_{A B}=0, \text { and } \bar{\eta}_{a}^{A} \bar{\eta}_{b}^{B} \mathcal{G}_{A B}=\bar{g}_{a b}
$$

where $\bar{\eta}_{a}^{A}$ are the components of the $N$ linearly independent vector fields in the same coordinates of the bulk where the components $\mathcal{G}_{A B}$ of the bulk metric are defined. The vectors $\left\{\overline{\mathcal{X}}_{, \mu}^{A}, \bar{\eta}_{a}^{B}\right\}$ define a Gaussian reference frame called here the embedding frame. The derivatives of the vectors $\bar{\eta}_{a}$ is expressed in terms of the second and third fundamental forms $\bar{k}_{\mu \nu a}, \bar{A}_{\mu a b}$ respectively by the Gauss-Weingerten equations [20]

$$
\bar{\eta}_{a, \alpha}^{A}=\bar{g}^{\mu \nu} \bar{k}_{\alpha \mu a} \overline{\mathcal{X}}_{, \nu}^{A}+\bar{g}^{m n} \bar{A}_{\alpha a m} \bar{\eta}_{n}^{A}
$$

Without loss of generality we may chose the normal vectors $\bar{\eta}_{a}$ to be orthogonal to each other, so that $\bar{g}_{a b}=\epsilon_{a} \delta_{a b}$, where $\epsilon_{a}= \pm 1$ depending on the signature of the bulk [19].

Nash's perturbative approach to embedding consists in subjecting the fundamental forms of $\bar{\sigma}_{4}$ to small parametric deviations along each normal vector. It can be also described by introducing a small perturbation with parameter $\delta y^{a}$, of the base vectors $\left\{\overline{\mathcal{X}}_{, \mu}^{A}, \eta_{a}^{A}\right\}$ along each normal $\bar{\eta}_{a}^{A}$ evaluated on $\bar{\sigma}_{4}$, obtaining another set of vectors (no sum on a)

$$
\begin{aligned}
\mathcal{Z}_{, \mu}^{A} & =\overline{\mathcal{X}}_{, \mu}^{A}+\left(\delta y^{a} £_{\bar{\eta}_{a}} \overline{\mathcal{X}}^{A}\right)_{, \mu}=\overline{\mathcal{X}}_{, \mu}^{A}-\delta y^{a}\left[\overline{\mathcal{X}}, \bar{\eta}_{a}\right]_{, \mu}^{A}=\overline{\mathcal{X}}_{, \mu}^{A}+\delta y^{a} \bar{\eta}_{a, \mu}^{A}, \\
\eta_{a}^{A} & =\bar{\eta}_{a}^{A}+\left(\delta y^{a} £_{\bar{\eta}_{a}} \bar{\eta}_{a}\right)^{A}=\bar{\eta}_{a}^{A}+\delta y^{a}\left[\bar{\eta}_{a}, \bar{\eta}_{a}\right]^{A}=\bar{\eta}_{a}^{A}
\end{aligned}
$$

\footnotetext{
${ }^{1}$ The perturbative approach to the embedding was originally proposed by J. E. Campbell in 1926. However, his result differs from Nash's theorem because analytic conditions where implicitly used 16, 17]. Since the perturbation procedure is based on regular and differentiable functions, the differentiable embedding is less restrictive to the geometry than the analytic embeddings.

${ }^{2}$ Capital Latin indices refer to the bulk, which is a Riemannian geometry with metric $\mathcal{G}_{A B}$ in arbitrary coordinates. Small case Latin indices refer to the extra dimensions going from 5 to D, and all Greek indices refer to the brane, from 1 to 4 . A curly $\mathcal{R}$ always denotes bulk curvatures, like in $\mathcal{R}_{A B C D}$. Ordinary capital $R$ like in $R_{\mu \nu}$ denotes brane-world curvatures. Covariant derivatives need to be specified, for the bulk or the brane-world metrics. For a vector $V^{A}$ in the bulk its covariant derivative with respect to $\mathcal{G}_{A B}$ is denoted as $V_{; B}^{A}$. On the other hand, from the point of view of the brane-world metric, the components $V^{A}$ behave as a set of $N$ scalar functions as in 20]. For generality we $\operatorname{denote} \mathcal{G}=\left|\operatorname{det}\left(\mathcal{G}_{A B}\right)\right|$.
} 
which define a perturbed embedding frame $\left\{\mathcal{Z}_{, \mu}^{A}, \eta_{a}^{A}\right\}$ in the bulk. Admitting that these new functions remain differentiable and regular and that they satisfy the equations similar to (2.1),

$$
\mathcal{Z}_{, \mu}^{A} \mathcal{Z}_{, \nu}^{B} \mathcal{G}_{A B}=g_{\mu \nu}, \quad \mathcal{Z}_{, \mu}^{A} \eta_{a}^{B} \mathcal{G}_{A B}=g_{\mu a}, \quad \eta_{a}^{A} \eta_{b}^{B} \mathcal{G}_{A B}=g_{a b}=\bar{g}_{a b}
$$

we obtain a $N$-parameter local family of submanifolds $\sigma_{4}$ generated by local perturbations of $\bar{\sigma}_{4}$, by a continuous variations of the parameters $\delta y^{a}$.

The next problem is to find a solution of these equations. However, instead of finding the coordinates $\mathcal{Z}^{A}$, it is more convenient to write the perturbed solution in terms of the fundamental forms, expressed in terms of the initial geometry of $\overline{\sigma_{4}}$. By direct substitution of $\mathcal{Z}_{, \mu}$ and $\eta_{a}^{A}$ derived from (2.3) in equations (2.5) we obtain

$$
\begin{aligned}
g_{\mu \nu}(x, y) & =\mathcal{Z}_{, \mu}^{A} \mathcal{Z}_{, \nu}^{B} \mathcal{G}_{A B}=\bar{g}_{\mu \nu}-2 \delta y^{a} \bar{k}_{\mu \nu a} \\
& +\delta y^{a} \delta y^{b}\left[\bar{g}^{\alpha \beta} \bar{k}_{\mu \alpha a} \bar{k}_{\nu \beta b}+g^{c d} \bar{A}_{\mu c a} \bar{A}_{\nu d b}\right], \\
g_{\mu a}(x, y) & =\mathcal{Z}_{, \mu}^{A} \eta_{a}^{B} \mathcal{G}_{A B}=\delta y^{b} A_{\mu a b}, \\
g_{a b}(x, y) & =\eta_{a}^{A} \eta_{b}^{B} \mathcal{G}_{A B}=\bar{g}_{a b} \\
k_{\mu \nu a}(x, y) & =-\eta_{a, \mu}^{A} \mathcal{Z}_{, \nu}^{B} \mathcal{G}_{A B} \\
& =\bar{k}_{\mu \nu a}-\delta y^{b} \bar{g}^{\alpha \beta} \bar{k}_{\mu \alpha a} \bar{k}_{\nu \beta b}-g^{c d} \delta y^{b} \bar{A}_{\mu c a} \bar{A}_{\nu d b}, \\
A_{\mu a b}(x, y) & =\eta_{a, \mu}^{A} \eta_{b}^{B} \mathcal{G}_{A B}=\bar{A}_{\mu a b}(x)
\end{aligned}
$$

The contravariant components of the perturbed geometry must be consistent with $\mathcal{G}^{A C} \mathcal{G}_{C B}=$ $\delta_{B}^{A}$, which can be realized by setting $g_{\mu \rho} g^{\rho \nu}=\delta_{\mu}^{\nu}, g_{a c} g^{c b}=\delta_{a}^{b}$. Since the indices $\mu$ and $b$ can never be equal, we must nave $g_{\mu \rho} g^{\rho b}+g_{\mu c} g^{c b}=\delta_{\mu}^{b}=0$. After some algebra we see that this corresponds to an identity $y^{m} y^{n} g^{a b} A_{\mu a m} A_{\nu b n}=-y^{b} y^{n} g^{m a} A_{\mu[a m]} A_{\nu[b n]} \equiv 0$.

Comparing (2.6) and (2.9) we obtain

$$
k_{\mu \nu a}=-\frac{1}{2} \frac{\partial g_{\mu \nu}}{\partial y^{a}}
$$

Consequently, the local bulk defined in a neighborhood around $\bar{\sigma}_{4}$, is foliated by this perturbed geometry, so that the Riemann curvature of the bulk may be expressed in the perturbed embedding frame. For any fields in the bulk $\xi$ and $\zeta$, the covariant derivative $D_{\xi} \zeta$ is defined by the metric affine connection $\Gamma_{A B C}$, with the Riemann tensor given by $\mathcal{R}(\xi, \zeta)=\left[D_{\xi}, D_{\zeta}\right]$. Writing the components of this tensor in the embedding frame we obtain the Gauss, Codazzi and Ricci equations, respectively:

$$
\begin{gathered}
\mathcal{R}_{A B C D} \mathcal{Z}_{, \alpha}^{A} \mathcal{Z}_{, \beta}^{B} \mathcal{Z}_{, \gamma}^{C} \mathcal{Z}_{, \delta}^{D}=R_{\alpha \beta \gamma \delta}-2 g^{m n} k_{\alpha[\gamma m} k_{\delta] \beta n} \\
\mathcal{R}_{A B C D} \mathcal{Z}_{, \alpha}^{A} \eta_{b}^{B} \mathcal{Z}_{, \gamma}^{C} \mathcal{Z}_{, \delta}^{D}=k_{\alpha[\gamma b ; \delta]}-g^{m n} A_{[\gamma m b} k_{\alpha \delta] n} \\
\mathcal{R}_{A B C D} \eta_{a}^{A} \eta_{b}^{B} \mathcal{Z}_{, \gamma}^{C} \mathcal{Z}_{, \delta}^{D}=-2 g^{m n} A_{[\gamma m a} A_{\delta] n b}-2 A_{[\gamma a b ; \delta]}-g^{\mu \nu} k_{[\gamma \mu a} k_{\delta] \nu b}
\end{gathered}
$$

which are the integrability conditions for the embedding. The differentiable embedding occurs when for a given Riemann tensor for the bulk these equations can be solved without appeal to analyticity. A substantial part of Nash's theorem consists in showing that the solution requires that the functions appearing in the right hand side must be regular. 
The expression (2.11) shows that besides the brane-world gravitational field the extrinsic curvature $k_{\mu \nu a}$ also propagate in the bulk. The implication of this is that the imposition of any restrictive conditions on $k_{\mu \nu a}$ also implies on restrictions on the propagation of the gravitational field of the brane-world. On the other hand, from (2.10) it follows that the third fundamental form $A_{\mu a b}$ does not propagate at all in the bulk, behaving as if it is a confined field.

The equations of motion of the brane-world follow directly from the Einstein-Hilbert principle on the bulk and from the integrability conditions (2.12)-(2.14). To see how this works take the trace of the first equation (2.5): $g^{\mu \nu} Z_{, \mu}^{A} Z_{, \nu}^{B} \mathcal{G}_{A B}=D-N=\mathcal{G}_{A B} \mathcal{G}^{A B}-g^{a b} g_{a b}$, and replace $g_{a b}$ from (2.5), obtaining

$$
g^{\mu \nu} Z_{, \mu}^{A} Z_{, \nu}^{B}=\mathcal{G}^{A B}-g^{a b} \eta_{a}^{A} \eta_{b}^{B}
$$

The contractions of (2.12) with $g^{\mu \nu}$, and using (2.15) gives the the Ricci tensor and Ricci scalar of the brane-world respectively expressed as

$$
R_{\mu \nu}=g^{c d}\left(g^{\alpha \beta} k_{\mu \alpha c} k_{\nu \beta d}-h_{c} k_{\mu \nu d}\right)+\mathcal{R}_{A B} Z_{, \mu}^{A} Z_{, \nu}^{B}-g^{a b} \mathcal{R}_{A B C D} \eta_{a}^{A} Z_{, \mu}^{B} Z_{, \nu}^{C} \eta_{b}^{D}
$$

After another contraction with $g^{\mu \nu}$, using again (2.15), and noting that $g^{a d} g^{b c} \mathcal{R}_{A B C D} \eta_{a}^{A} \eta_{b}^{B} \eta_{c}^{C} \eta_{d}^{D}=0$, we obtain the Ricci scalar

$$
R=\left(K^{2}-h^{2}\right)+\mathcal{R}-2 g^{a b} \mathcal{R}_{A B} \eta_{a}^{A} \eta_{b}^{B}
$$

where $K^{2}=g^{a b} k^{\mu \nu}{ }_{a} k_{\mu \nu b} . h_{a}=g^{\mu \nu} k_{\mu \nu a}$ and $h^{2}=g^{a b} h_{a} h_{b}$. Therefore the Einstein-Hilbert action for the bulk geometry in $D$-dimensions can be written as

$$
\begin{array}{r}
\mathcal{A}=\int \mathcal{R} \sqrt{\mathcal{G}} d^{D} v=\int\left[R-\left(K^{2}-h^{2}\right)+2 g^{a b} \mathcal{R}_{A B} \eta_{a}^{A} \eta_{b}^{B}\right] \sqrt{\mathcal{G}} d^{D} v \\
=\alpha_{*} \int \mathcal{L}^{*} \sqrt{\mathcal{G}} d^{D} v
\end{array}
$$

where $\alpha_{*}$ denotes the fundamental energy scale in the bulk and $\mathcal{L}^{*}$ is the source Lagrangian. The Euler-Lagrange equations of (2.18) with respect to $\mathcal{G}_{A B}$ are Einstein's equations in $D$ dimensions:

$$
\mathcal{R}_{A B}-\frac{1}{2} \mathcal{R} \mathcal{G}_{A B}=\alpha_{*} T_{A B}^{*}
$$

Here $T_{A B}^{*}$ denote the components of the energy-momentum tensor of the sources.

The equations of motion of the embedded brane-world can be derived directly from the components of (2.19) written in the embedding frame. The tangent components follow from the contractions of $(2.19)$ with $\mathcal{Z}_{, \mu}^{A} \mathcal{Z}_{, \nu}^{B}$. After using (2.16) and (2.17) we obtain

$$
R_{\mu \nu}-\frac{1}{2} R g_{\mu \nu}-Q_{\mu \nu}-W_{\mu \nu}-g^{a b} \mathcal{R}_{A B} \eta_{a}^{A} \eta_{b}^{B}=\alpha_{*} T_{\mu \nu}^{*}
$$

where we have denoted

$$
\begin{aligned}
Q_{\mu \nu} & =g^{a b} k_{\mu a}^{\rho} k_{\rho \nu b}-g^{a b} h_{a} k_{\mu \nu b}-\frac{1}{2}\left(K^{2}-h^{2}\right) g_{\mu \nu} \\
W_{\mu \nu} & =g^{a d} \mathcal{R}_{A B C D} \eta_{a}^{A} \mathcal{Z}_{, \mu}^{B} \mathcal{Z}_{, \nu}^{C} \eta_{d}^{D}
\end{aligned}
$$


By a direct calculation we can see that the extrinsic tensor $Q_{\mu \nu}$ is an independently conserved quantity with respect to the brane-world metric.

The contraction of $(2.19)$ with $\mathcal{Z}_{\mu}^{A} \eta_{b}^{B}$ gives a vectorial equation. Using (2.17) and (2.13) we obtain

$$
k_{\mu a ; \rho}^{\rho}-h_{a, \mu}+A_{\rho c a} k_{\mu}^{\rho c}-A_{\mu c a} h^{c}+2 W_{\mu a}=-2 \alpha_{*}\left(T_{\mu a}^{*}-\frac{1}{N+2} T^{*} g_{\mu a}\right)
$$

where we have denoted

$$
W_{\mu a}=g^{b d} \mathcal{R}_{A B C D} \eta_{a}^{A} \eta_{b}^{B} \mathcal{Z}_{, \mu}^{C} \eta_{d}^{D}
$$

Finally, contracting (2.19) with $\eta_{a}^{A} \eta_{b}^{B}$ we obtain $N(N+1) / 2$ scalar equations involving the so called Hawking-Gibbons term $S_{a b}=\mathcal{R}_{A B} \eta_{a}^{A} \eta_{b}^{B}$ and its trace $S=g^{a b} S_{a b}$

$$
S_{a b}-S g_{a b}-\frac{1}{2}\left[R-K^{2}+h^{2}\right] g_{a b}=\alpha_{*} T_{a b}^{*}
$$

In its most general form, without assuming extra dimensional matter, the confinement hypothesis states that the only non-vanishing components of $T_{A B}$ are the tangent components $T_{\mu \nu}$ representing the confined sources [2]. Therefore we set

$$
\begin{aligned}
& \alpha_{*} T_{A B}^{*} \mathcal{Z}_{, \mu}^{A} \mathcal{Z}_{, \nu}^{B}=\alpha_{*} T_{\mu \nu}^{*}=-8 \pi G T_{\mu \nu} \\
& \alpha_{*} T_{A B}^{*} \mathcal{Z}_{, \mu}^{A} \eta_{a}^{B}=\alpha_{*} T_{\mu a}^{*}=0 \\
& \alpha_{*} T_{A B}^{*} \eta_{a}^{A} \eta_{b}^{B}=\alpha_{*} T_{a b}^{*}=0
\end{aligned}
$$

Equations (2.20), (2.22) and (2.24) with confinement conditions are sometimes called the gravi-tensor, gravi-vector and gravi-scalars (Usually a single gravi-scalar equation in the 5-dimensional models [21]) equations respectively. These represent generalizations of Einstein's equations of general relativity, in the sense that they describe the evolution of all geometrical components $g_{\mu \nu}, A_{\mu a b}$ and $k_{\mu \nu a}$ of the brane-world. Clearly, the usual Einstein's equations are recovered when all elements of the extrinsic geometry are removed from those equations.

\section{Canonical Equations}

The standard ADM canonical quantization of the gravitational field in general relativity was originally intended to describe the quantum fluctuations of 3-dimensional hypersurfaces in a space-time [22]. The space-time metric is decomposed in 3-surface components, plus a shift vector and a lapse function defined in a Gaussian reference frame defined on the 3dimensional hypersurface. After writing the Einstein-Hilbert Lagrangian in this Gaussian frame, the Euler-Lagrange equations with respect to the shift leads to the vanishing of the Hamiltonian. This is not a real problem because in principle the system could be solved by use of Dirac's standard procedure for constrained systems. However, as it is well known, the Poisson bracket structure does not propagate covariantly as it would be expected. In spite of all efforts made up to the present, this problem remains unsolved [23, 24, 25, 26]. It is possible to describe a non-constrained canonical system in a special frame defined by a 
3-dimensional hypersurface orthogonal Gaussian coordinate system. In such special frame the shift vector vanishes and the Hamiltonian constraint does not apply [27]. Nonetheless, this has been regarded as of little value for general relativity itself, essentially because the diffeomorphism group of the theory is one of the fundamental postulates of the theory [28].

The extension of the ADM canonical formulation to the brane-world is straightforward but it requires a few adaptations: First, the bulk is locally foliated by a continuous sequence of brane-worlds propagating along the extra dimensions rather than by a 3 -surface propagating along a single time direction. Secondly, the confinement hypothesis implies that the diffeomorphism invariance do not extend to the extra dimensions, otherwise a coordinate transformation in the bulk would have the effect of introducing a component of the energy-momentum tensor of the confined fields and ordinary matter in the bulk. Therefore, in order to maintain the intended solution of the hierarchy problem, the diffeomorphism of the brane-world must be restricted as a confined symmetry. Actually this can be regarded as one of the merits of brane-world theory, which differentiates it from being just a higher dimensional version of general relativity. However, to deserve such merit the extra dimensions need to be taken seriously as true physical degrees of freedom in the canonical formulation of the theory. The momentum conjugated to the metric field $\mathcal{G}_{A B}$, with respect to the displacement along $\eta_{a}$ is defined as usual

$$
p^{A B}{ }_{(a)}=\frac{\partial \mathcal{L}}{\partial\left(\frac{\partial \mathcal{G}_{A B}}{\partial y^{a}}\right)}
$$

where $\mathcal{L}$ is the Einstein-Hilbert Lagrangian of the bulk in (2.18). Noting that in the embedding frame we can write $2 g^{a b} \mathcal{R}_{A B} \eta_{a}^{A} \eta_{b}^{B}=K^{2}-g^{a b} h_{a, b}$, after eliminating the divergence term $g^{a b} h_{a, b}$, the Lagrangian can be simplified to

$$
\mathcal{L}=\left[R+\left(K^{2}+h^{2}\right)\right] \sqrt{\mathcal{G}}
$$

Therefore, using (2.11) we obtain the canonically conjugated momenta

$$
\begin{aligned}
& p_{(a)}^{\mu \nu}=\frac{\partial \mathcal{L}}{\left(\frac{\partial g_{\mu \nu}}{\partial y^{a}}\right)}=-\frac{1}{2} \frac{\partial \mathcal{L}}{\partial k_{\mu \nu a}}=-\left(k^{\mu \nu}{ }_{a}+h_{a} g^{\mu \nu}\right) \sqrt{\mathcal{G}} \\
& p_{(b)}^{\mu a}=\frac{\partial \mathcal{L}}{\left(\frac{\partial g_{\mu a}}{\partial y^{b}}\right)}=\frac{\partial \mathcal{L}}{\partial A_{\mu a b}}=0, \quad p^{a b}{ }_{(c)}=\frac{\partial \mathcal{L}}{\left(\frac{\partial g_{a b}}{\partial y^{c}}\right)}=0
\end{aligned}
$$

The last two components are equal to zero because the Lagrangian (3.1) does not depend explicitly on $A_{\mu a b}$ and on $g_{a b, c}$.

Using the above expressions, the Hamiltonian $\mathcal{H}_{a}$ corresponding to the displacement along each orthogonal direction $\eta_{a}$ separately, follows from a partial Legendre transformation (no sum on (a)):

$$
\mathcal{H}_{a}\left(g, p_{(a)}\right)=p^{A B}{ }_{(a)} g_{A B, a}-\mathcal{L}=-R \sqrt{\mathcal{G}}-\left[\left(K^{2}+h^{2}+2\left(K_{a}^{2}+h_{a}^{2}\right)\right] \sqrt{\mathcal{G}}\right.
$$


where we have denoted $K_{a}^{2}=k_{a}^{\mu \nu} k_{\mu \nu a}, K^{2}=g^{a b} K_{a} K_{b}$, and $p_{(a)}=g^{\mu \nu} p_{\mu \nu(a)}$. After replacing $h_{a}=\frac{-p_{(a)}}{5 \sqrt{\mathcal{G}}}$ it follows that

$$
\mathcal{H}_{a}=-R \sqrt{\mathcal{G}}-\frac{1}{\sqrt{\mathcal{G}}}\left[\frac{3 p^{2}}{5}+2 \frac{p_{(a)}^{2}}{5}+p_{(a)}^{\mu \nu} p_{\mu \nu(a)}\right]
$$

For a given functional $\mathcal{F}\left(g_{\mu \nu}, p_{\mu \nu}\right)$ defined in the phase space of the brane-world, the propagation of $\mathcal{F}$ along $y^{a}$ is given by the Poisson brackets with each Hamiltonian separately

$$
\frac{\tilde{\delta} \mathcal{F}}{\tilde{\delta} y^{a}}=\left[\mathcal{F}, \mathcal{H}_{a}\right]=\frac{\tilde{\delta} \mathcal{F}}{\tilde{\delta} g_{\mu \nu}} \frac{\tilde{\delta} \mathcal{H}_{a}}{\tilde{\delta} p^{\mu \nu(a)}}-\frac{\tilde{\delta} \mathcal{F}}{\tilde{\delta} p^{\mu \nu(a)}} \frac{\tilde{\delta} \mathcal{H}_{a}}{\tilde{\delta} g_{\mu \nu}}
$$

Here $\tilde{\delta}$ denotes the standard functional derivative in phase space.

Hamilton's equations for the brane-world with respect to each extra coordinate $y^{a}$ may be written as

$$
\begin{aligned}
& \frac{d g_{\mu \nu}}{d y^{a}}=\frac{\tilde{\delta} \mathcal{H}_{a}}{\tilde{\delta} p^{\mu \nu(a)}}=\left[g_{\mu \nu}, \mathcal{H}_{a}\right]=-2 k_{\mu \nu a}, \\
& \frac{d p_{(a)}^{\mu \nu}}{d y^{a}}=-\frac{\tilde{\delta} \mathcal{H}_{a}}{\tilde{\delta} g_{\mu \nu}}=\left[p_{(a)}^{\mu \nu}, \mathcal{H}_{a}\right]
\end{aligned}
$$

As it can be seen, the differences between the brane-world canonical formulation and the $\mathrm{ADM}$ formulation of general relativity follow from the non-vanishing of the Hamiltonians $\mathcal{H}_{a}$, as a consequence of the brane-world scheme for solving the hierarchy problem. With this result, the ADM quantization program can be retaken, with the difference that the quantum equation should describe the "states" of four-dimensional submanifolds in the bulk, with respect to the extra dimensions.

\section{Tomonaga-Schwinger Quantum States}

The Tomonaga-Schwinger equation originated from Dirac's many fingered time formalism for relativistic quantum theory, in which a set of $N$ electrons was associated to $N$ proper times satisfying $N$ Schrodinger's-like equations [29]. The continuous limit of this equation was formulated by Tomonaga for a relativistic field defined in a region of space-time characterized by an evolving space-like 3-hypersurface $\sigma$ with a time direction attached to each of its point. This geometric extension of Dirac's many fingered time, which was soon realized to be equivalent to the interaction representation of quantum mechanics developed by Schwinger [30, 31, 32]. Here, it is more convenient to look at the Tomonaga-Schwinger equation from the geometrical point of view written as

$$
i \hbar \frac{\delta \Psi}{\delta \sigma}=\hat{\mathcal{H}}_{\sigma} \Psi
$$

which represents a generalization of Schrodinger's equation, describing the quantum state functional $\Psi$ of a space-like 3-hypersurface $\sigma$ embedded in Minkowski space-time. In the 
right hand side, the Hamiltonian operator describes the translational operator along a timelike direction orthogonal to $\sigma$. The functional derivative in the left hand side is defined by the limit

$$
\frac{\delta \Psi}{\delta \sigma}=\lim _{\Delta V \rightarrow 0} \frac{\Psi\left(\sigma^{\prime}\right)-\Psi(\sigma)}{\Delta V}
$$

where $\Delta V$ denotes the local volume element between two neighboring hypersurfaces $\sigma$ and $\sigma^{\prime}$.

The main difficulty of (4.1) is that it is not easily integrable. In the particular case where $\left[\hat{\mathcal{H}}_{\sigma}, \hat{\mathcal{H}}_{\sigma^{\prime}}\right]=0$, the equation can be integrated, but the hypersurfaces $\sigma$ and $\sigma^{\prime}$ are necessarily flat. In the general case where the hypersurfaces are not flat, the solutions of (4.1) can be determined as an approximate solution after the application of the YangFeldmann formalism and Dyson's expression for the $S$ matrix [32, 33]. The difficulty in solving (4.1) can be traced back to the fact that the limit operation in (4.2) was not defined. In fact, the conditions to decide how close $\sigma$ and $\sigma^{\prime}$ are were not given previously, and it can be decided only after solving the quantum equation itself using some quantum approximation method.

In the application of (4.1) to the brane-world, the limit operation between two fourdimensional brane-worlds $\sigma_{4}$ and $\sigma_{4}^{\prime}$ is improved because Nash's theorem shows at the classical level how to tell the separation between the two sub manifolds. In other words, since each brane-world was generated by classical perturbations of an initial sub manifold $\sigma_{4}$, the volume element in (4.2) has been already specified by the parameter $\delta y^{a}$ of the perturbed geometry. In practice, we may split the bulk volume $\Delta V$ between $\sigma_{4}$ and $\sigma_{4}^{\prime}$ into a product of the volume $\Delta v$ of a a small compact region in $\sigma_{4}$, times the variation $\Delta y^{a}$ of the extra dimensional coordinates $y^{a}$. Therefore, it sufficient to specify only the limit operation $\Delta y^{a} \rightarrow 0$ and the functional derivative (4.2) for density functions with compact support on the brane-world, with respect to each extra dimension can be simplified to

$$
\left.\frac{\delta \Psi}{\delta \sigma}\right\rfloor_{y^{a}}=\lim _{\Delta y^{a} \rightarrow 0} \frac{\Psi\left(\sigma^{\prime}\right)-\Psi(\sigma)}{\Delta y^{a}}=\frac{\partial \Psi}{\partial y^{a}}
$$

Repeating for all extra dimensions, we find that the Tomonaga-Schwinger equation (4.1) can be extended the brane-world, as a system of $N$ partial equations, one for each extra dimension

$$
i \hbar \frac{\partial \Psi_{a}}{\partial y^{a}}=\hat{\mathcal{H}}_{a} \Psi_{a}, \quad a=5 . . D
$$

which gives to $\hat{\mathcal{H}}_{a}$ the interpretation of the extra dimensional translational operator. The final quantum state is given by the superposition of the $N$ separates states $\Psi_{a}$ as $\Psi=\sum B^{a} \Psi_{a}$. The state functional density $\Psi$ represents the quantum fluctuation of the brane-world sub manifold in the bulk at the (Tev) energy scale, subjected to quantum uncertainties and a state probability given by

$$
\|\Psi\|^{2}=\int \Psi^{\dagger} \Psi \delta y \delta v
$$


An observer confined to the brane-world may evaluate the quantum expectation values of the brane-world metric and the extrinsic curvature are given by

$$
<\Psi\left|\hat{g}_{\mu \nu}\right| \Psi>=\int \Psi^{\dagger} \hat{g}_{\mu \nu} \Psi \delta y \delta v, \quad<\Psi\left|\hat{k}_{\mu \nu}\right| \Psi>=\int \Psi^{\dagger} \hat{k}_{\mu \nu} \Psi \delta y \delta v
$$

Since the classical $k_{\mu \nu a}$ is the derivative of the metric $g_{\mu \nu}$ with respect to $y_{a}$, we may set boundary conditions on these quantities at the initial brane-world $y^{a}=0$ to determine the final solution.

\section{Overview and Perspectives}

We have shown that the Einstein-Hilbert principle applied to the bulk geometry plus the differentiable embedding conditions are sufficient to determine the classical and quantum structures of the brane-world in D-dimensions. In particular, it was shown that Nash's theorem makes it possible to generate any embedded sub manifold by a continuous sequence of infinitesimal perturbations of an arbitrarily given embedded geometry along the extra dimensions. Using the classical perturbative embeddings, and the basic principles of the brane-world theory we have obtained a canonical structure very much like the ADM canonical formulation of general relativity, with the exception that the Hamiltonians do not vanish.

The definition of the functional derivatives was improved with respect to four-dimensional field theory, by using the previously defined perturbative embedding structure of the braneworld. The quantization of the brane-world was described the Tomonaga-Schwinger equation defined for brane-world sub manifolds, calculated for each extra dimension. Actually, as a result of the the the classical perturbation theory, the Tomonaga-Schwinger equation becomes exactly integrable.

In view of current astrophysical observations and the near future high energy experiments, there are some applications of the quantum brane-world theory to be detailed in subsequent papers:

(a) Brane-world Cosmology

Brane-world cosmology offers a possible explanation to the accelerated expansion of the universe, resulting from the modification of the Friedman's equation by the presence of the extrinsic curvature included in the tensor $Q_{\mu \nu}$ given by (2.21) [10]. The presence of this tensor has the meaning that the brane-world vacuum is more complex than the vacuum in general relativity. In fact, for a constant curvature bulk with curvature $\Lambda_{*}$, after eliminating redundant terms, the gravi-tensor vacuum equation becomes $R_{\mu \nu}-1 / 2 R g_{\mu \nu}-Q_{\mu \nu}+\Lambda_{*} g_{\mu \nu}=$ 0 .

Therefore, the vacuum energy density $<\rho_{v}>$ resulting from gravitationally coupled fields must be revaluated, including the extrinsic curvature component. This suggests that in some epochs, say at the early inflationary period, the extrinsic curvature may contribute to the vacuum energy, differently from other periods.

The particular case where we have only one extra dimensions $(D=5)$, has some limitations with respect to the differentiable embedding. However, some cosmological models like 
the FRW, deSitter and anti deSitter solutions of Einstein's equations in four dimensions can be embedded in five dimensional bulks without restrictions, in accordance with the perturbative embedding equations previously shown. Consequently, in such brane-world cosmological models the conditions required for a proper definition of the functional derivatives in the Tomonaga-Schwinger equation are well established, and the equation can can be integrated without difficulty.

(b) Laboratory production of mini black holes:

Brane-world gravity predict the generation of short lived mini black holes produced at the Tev energy in the laboratory, resulting from proton-proton collisions [34]. However, using semi-classical quantum gravity in four dimensions, we have learned that quantum unitarity does not necessarily hold true during the black hole evaporation. On the other hand, using Euclidean path integral, it was shown that the unitarity can be restored with the aid of the ADS/CFT correspondence in the framework of $A d S_{5} \times S^{5}$ string theory [35].

Since the generation of mini black holes are possible only in the brane-world context, the whole process includes the original Minkowski's space-time where the experiment is devised. Soon after the collision, the space-time must be deformable into a Schwarzschild or a Reissner-Nordstrom black hole. Finally, after a short period of evaporation the spacetime may be back to Minkowski's configuration or else leaves a curved remnant. The description of such process can start with the classical perturbations in accordance with Nash's embedded geometries, but the unitarity is has to be decided at the quantum level. In this respect we notice that both Schwarzschild and Reissner-Nordstrom black holes are well defined submanifolds embedded in a six-dimensional flat bulk with signature $(4,2)$. In this case, the bulk isometry group $S O(4,2)$ is isomorphic to the conformal group in Minkowski's space-time, compatible with the ADS/CFT correspondence adapted to the brane-world [36]. Therefore, the quantum unitarity implicitly assumed in the TomonagaSchwinger equation, must be consistent with the black hole evaporation theorems in six dimensions.

(c) Quantum Four-manifold Theory:

The above description of quantum theory of the brane-world is based almost entirely on the general theory of differentiable sub manifolds. This suggests a quantum theory of four-dimensional sub manifolds. It starts with the classical perturbations of embedded geometries, but ends with a quantum version of the embedding theorem, including the fluctuations of the embedding as described by the Tomonaga-Schwinger equation. This quantum theory of submanifolds would be particularly interesting when the bulk has dimensions greater than five, where the third fundamental form behaves similarly to a gauge field with respect to the extra dimensional group of isometries. The identification of the third fundamental form as a gauge field with the symmetry of the extra dimensions plying the role of the gauge group is old, but it was never taken seriously [37].

One frequent criticism to string theory is that it depends on a pre-existing background space-time with 10 (or 11) dimensions, acting as the host space for all possible dynamics [38, 39]. On the other hand, loop quantum gravity does not require such background, but it depends on a previously existing spin network structure [40]. Quantum brane-world 
gravity does not have a background space in the same sense of string theory because the bulk is the primary dynamical object. The Einstein-Hilbert principle applied to the bulk geometry provides all dynamics of the brane-world, without requiring any new algebraic structure besides the theory of differentiable manifolds, where our basic notions of space, topology and analysis begin and make sense.

\section{References}

[1] C. Misner, Rev. Mod. Phys. $\underline{29}, 497$ (1957).

[2] N. Arkani-Hamed et Al, Phys. Lett. B429,263 (1998), hep-ph/9807344 Phys. Rev. Lett. $\underline{84}$, $586,(2000)$

[3] I. Antoniadis et al, Phys. Lett. ibid B246, 377, (1990); $\underline{B 436}, 257$ (1998).

[4] K. Akama, Pregeometry, in Gauge Theory and Gravitation, Lecture notes in Physics 176 (Springer Verlag (1983)), hep-th/0001113;

[5] V. A. Rubakov \& M. E. Shaposhnikov, Phys. Lett. 125B, 136, (1983);

[6] T. Regge \& C. Teitelboim, General Relativity a la String, proc. II Marcel Grossmann meeting, ICTP, Trieste (1972).

[7] M. Visser, Phys. Lett. 159B, 22 (1985), hep-th/9910093.

[8] P. Horava \& E. Witten, Nuc. Phys. $\underline{\text { B475 }}$, 94 (1996)

[9] G. Landsberg, J.Phys. G32, R337, (2006), hep-ph/0607297

[10] M. D. Maia et al, Class.Quant.Grav. 22, 1623 (2005), astro-ph/0403072

[11] L. Randall \& R. Sundrum, Phys. Rev. Lett. $\underline{83}, 4690$ (1998); ibid $\underline{83}, 3370$ (1999)

[12] K. Maeda, Phys. Rev. D64, 123525 (2001). astro-ph/0012313,

[13] A. Friedman, Rev. Mod. Phys. 37, 201, 1965

[14] M. Janet, Ann. Soc. Pol. Mat $\underline{5}$, (1928)

[15] E. Cartan, Ann. Soc. Pol. Mat $\underline{6}, 1$, (1927)

[16] J. E. Campbell, A course of Differential Geometry, Claredon Press, Oxford (1926)

[17] F. Dahia, C. Romero, Braz.J.Phys. 포, 1140, (2005)

[18] J. Nash, Ann. Maths. $\underline{63}, 20$ (1956)

[19] R. Greene, Memoirs Amer. Math. Soc. 97, (1970)

[20] L.P. Eisenhart, Princeton U. P. Sixth print, p.159 ff, (1966).

[21] R. Durrer \& P. Kocian, Class. Quant. Grav. 21, 2127, (2004), hep-th/030518

[22] R. Arnowitt, S. Deser and C. Misner, The Dynamics of General Relativity. In Gravitation: An introduction to Current Research, L. Witten, Ed. John Willey \& Sons, p. 227, (1962)

[23] K. V. Kuchar, Canonical Quantum Gravity, $13^{\text {th }}$ Intl. Conf. on General Relativity \& Gravitation, Cordoba, Argentina (1992). Ibid. Time and Interpretations of Quantum Gravity $4^{\text {th }}$ Can. Conf. on general Relativity and relati. Astrophys. World. Scientific (1991). 
[24] C. J. Isham. Canonical Quantization and the Problem of Time NATO Advanced Studies Institute, Salamanca (1992),

[25] S. Hojman et al, Ann. of Phys. $\underline{96}, 88,(1976)$.

[26] E. Alvarez, Lect.Notes Phys. $\underline{669}, 31-58$ (2005), gr-qc/0405107

[27] P. A. M. Dirac Phys. Rev. 114, 924, (1959)

[28] P A. M. Dirac Proc.Roy.Soc.Lond. A246, 333, (1958).

[29] P. A. M. Dirac, Proc. Roy. Soc. London $\underline{136}, 453$ (1932)

[30] S. Tomonaga, Prog. Theor. Phys. 1 , 27 (1946)

[31] J. Schwinger, Phys. Rev. $\underline{74}, 1439$ (1948)

[32] K. Nishijima, Fields and Particles, W. A. Benjamin, (1969).

[33] W. Pauli, Selected Topics in Field Quantization, MIT Press, (1973); Dover edition (1973), p. 161

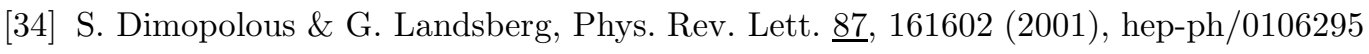

[35] S. Hawking, Phys. Rev. D72, 084013 (2005), hep-th/0507171

[36] M. D. Maia, Int.J.Mod.Phys. D14, 2251, (2005), gr-qc/0505119

[37] Y. Ne'emann, Rev. Mod. Phys., 37, 227 (1965)

[38] A. Ashtekar \& Jerzy Lewandowski, Class.Quant.Grav.21, R53(2004), gr-qc/0404018

[39] T. Thiemann, AEI-2006-066, hep-th/0608210

[40] H. Nicolai, K. Peeters \& M. Zamaklar, hep-th/0501114 